\title{
RELATIVELY FREE INVARIANT ALGEBRAS OF FINITE REFLECTION GROUPS
}

\author{
MÁTYÁS DOMOKOS
}

\begin{abstract}
Let $G$ be a finite subgroup of $G l_{n}(K)$ ( $K$ is a field of characteristic 0 and $n \geq 2$ ) acting by linear substitution on a relatively free algebra $K\left\langle x_{1}, \ldots, x_{n}\right\rangle / I$ of a variety of unitary associative algebras. The algebra of invariants is relatively free if and only if $G$ is a pseudo-reflection group and $I$ contains the polynomial $\left[\left[x_{2}, x_{1}\right], x_{1}\right]$.
\end{abstract}

\section{INTRODUCTION}

Throughout the paper $K$ is a field of characteristic 0 and $K\left\langle x_{1}, \ldots, x_{n}\right\rangle$ is the unitary free associative $K$-algebra of rank $n$. The general linear group $G l_{n}=$ $G l_{n}(K)$ acts on the free algebra by linear substitution. More explicitly, if $g=$ $\left(g_{i j}\right) \in G l_{n}$, then

$$
g \cdot x_{j}=\sum_{i=1}^{n} g_{i j} x_{i},
$$

and for any $f\left(x_{1}, \ldots, x_{n}\right) \in K\left\langle x_{1}, \ldots, x_{n}\right\rangle$

$$
g \cdot f=f\left(g \cdot x_{1}, \ldots, g \cdot x_{n}\right) .
$$

An ideal $I$ of the unitary free associative algebra $K\left\langle x_{1}, x_{2}, \ldots\right\rangle$ of countable rank is called a $T$-ideal if $I$ is invariant under any $K$-algebra endomorphism of the free algebra, that is, $f\left(x_{1}, \ldots, x_{n}\right) \in I$ implies $f\left(u_{1}, \ldots, u_{n}\right) \in I$ for any $u_{1}, \ldots, u_{n} \in$ $K\left\langle x_{1}, x_{2}, \ldots\right\rangle$.

$I_{n}=I \cap K\left\langle x_{1}, \ldots, x_{n}\right\rangle$ is invariant under the action of $G l_{n}$, so

$$
F_{n}(I)=K\left\langle x_{1}, \ldots, x_{n}\right\rangle / I_{n}
$$

inherits the $G l_{n}$-structure. $F_{n}(I)$ is a relatively free algebra of rank $n$ of the variety of unitary associative algebras satisfying all the identities $f=0$ with $f \in I$. Denote by $y_{i}$ the image of $x_{i}$ under the natural homomorphism

$$
K\left\langle x_{1}, \ldots, x_{n}\right\rangle \rightarrow K\left\langle x_{1}, \ldots, x_{n}\right\rangle / I_{n} .
$$

Received by the editors September 7, 1994.

1991 Mathematics Subject Classification. Primary 16W20; Secondary 16R10.

Key words and phrases. Relatively free algebra, reflection group, invariants, Hilbert series.

This research was partially supported by Széchenyi István Scholarship Foundation and by Hungarian National Foundation for Scientific Research grant no. T4265. 
Let $G$ be a finite subgroup of $G l_{n}$ and denote by

$$
F_{n}(I)^{G}=\left\{f \in F_{n}(I) \mid g \cdot f=f \forall g \in G\right\}
$$

the algebra of invariants of $G$. We refer to the article of Formanek [8] for a survey of the results on the algebra of invariants of a finite linear group acting on a relatively free algebra. In the special case of the variety of all unitary commutative algebras, that is, when $I$ is generated by the commutator $\left[x_{1}, x_{2}\right]=x_{1} x_{2}-x_{2} x_{1}$, the relatively free algebra

$$
F_{n}(I)=K\left[x_{1}, \ldots, x_{n}\right]
$$

is the $n$ variable commutative polynomial algebra, and the study of $F_{n}(I)^{G}$ is the topic of classical invariant theory.

An element $g \in G l_{n}(K)$ is called a pseudo-reflection, if it fixes an $n-1$ dimensional subspace of $K^{n}$. If $g$ is of finite order then $g$ is a pseudo-reflection if and only if $g$ has the eigenvalue 1 with multiplicity $n-1$. A finite subgroup $G<G l_{n}$ is called a pseudo-reflection group if it is generated by pseudo-reflections. Our starting point is the following famous result (Shephard-Todd [13], Chevalley [2]):

Theorem 1.1. $K\left[x_{1}, \ldots, x_{n}\right]^{G}$ is a polynomial algebra if and only if $G$ is a pseudoreflection group. Moreover, if $G$ is a pseudo-reflection group, then there exist $n$ algebraically independent homogeneous forms which generate $K\left[x_{1}, \ldots, x_{n}\right]^{G}$. The degrees $d_{1} \leq \ldots \leq d_{n}$ of these forms are uniquely determined by $G$, and are called the degrees of $G$. We have the equality $|G|=\prod_{i=1}^{n} d_{i}$, and the number of pseudoreflections in $G$ is $\sum_{i=1}^{n}\left(d_{i}-1\right)$.

We shall extend the notion of algebraic independence to any variety of unitary associative $K$-algebras. We fix a T-ideal $I$, and denote by $\mathcal{V}(I)$ the variety defined by $I$. Let $R$ be an algebra in $\mathcal{V}(I)$. We say that the elements $f_{1}, \ldots, f_{m} \in R$ are algebraically independent, if $h\left(f_{1}, \ldots, f_{m}\right)=0$ for some $h \in K\left\langle x_{1}, \ldots, x_{m}\right\rangle$ implies that $h \in I$. Note that this notion of algebraic independence depends on the T-ideal $I$ and belongs to the variety $\mathcal{V}(I)$.

Guralnick proved in [9] that if $n \geq 2, k \geq 2$ and $I$ is the T-ideal of the $k \times k$ matrix algebra over $K$, then $F_{n}(I)^{G}$ is not relatively free for any finite group $G$. As it was pointed out in $\left[8\right.$, p. 105], this result implies easily that $F_{n}(I)^{G}$ is not relatively free if $I$ is contained in the T-ideal of the $2 \times 2$ matrix algebra. In this paper we give a complete answer to the question of determining when $F_{n}(I)^{G}$ is relatively free. The answer was conjectured by Drensky [7].

The case $n=1$ is trivial, because then $F_{1}(I) \cong K\left[x_{1}\right] \cong K\left\langle x_{1}\right\rangle$ and $G$ is a cyclic group acting by scalar multiplication, so the algebra of invariants is $K\left[x^{m}\right]$ for some positive integer $m$.

Theorem 1.2. Let $G<G l_{n}(K)(n \geq 2)$ be a finite group and let $I$ be a T-ideal. Then $F_{n}(I)^{G}$ is generated by algebraically independent elements if and only if $G$ is a pseudo-reflection group and I contains the polynomial $\left[\left[x_{2}, x_{1}\right], x_{1}\right]$.

\section{Preliminaries}

The algebra $K\left\langle x_{1}, \ldots, x_{n}\right\rangle^{G}$ is always free and almost never finitely generated by [4] and [10]. However, working in a proper subvariety of the variety of all unitary associative $K$-algebras the notion of transcendence degree makes sense. 
Proposition 2.1. Fix a non-zero T-ideal $I$ and consider the variety $\mathcal{V}(I)$. If $R$ is an algebra in the variety $\mathcal{V}(I)$ generated by $n$ elements, then it does not contain more than $n$ algebraically independent elements.

Proof. $R$ is a homomorphic image of $F_{n}(I)$ and obviously the preimages of algebraically independent elements of $R$ are also algebraically independent, so it suffices to prove the proposition for the relatively free algebra $F_{n}(I)$. Assume that $f_{1}, \ldots, f_{m} \in F_{n}(I)$ are algebraically independent. Let $J \subseteq K\left\langle x_{1}, x_{2}, \ldots\right\rangle$ be the radical of $I$, that is,

$$
J=\left\{f \in K\left\langle x_{1}, x_{2}, \ldots\right\rangle \mid f^{N} \in I \text { for some } N\right\} .
$$

It is well known (see for example [12, Theorems 1.5.32, 2.4.7 and 3.2.6]) that $J$ is the set of identities of the $k \times k$ matrix algebra for some $k \geq 1$. We have the natural onto homomorphism

$$
K\left\langle x_{1}, \ldots, x_{n}\right\rangle / I_{n} \rightarrow K\left\langle x_{1}, \ldots, x_{n}\right\rangle / J_{n} .
$$

Denote by $h_{1}, \ldots, h_{m}$ the images of $f_{1}, \ldots, f_{m}$. Suppose that for some $p \in$ $K\left\langle x_{1}, \ldots, x_{m}\right\rangle$ we have $p\left(h_{1}, \ldots, h_{m}\right)=0$. Then there exists an integer $N$ such that $p^{N}\left(f_{1}, \ldots, f_{m}\right)=0$ in $F_{n}(I)$, and the algebraic independence of the $f_{i}$ s implies that $p^{N}\left(x_{1}, \ldots, x_{m}\right) \in I_{m}$. Hence $p\left(x_{1}, \ldots, x_{m}\right) \in J_{m}$ by the definition of $J_{m}$, and this shows the algebraic independence of $h_{1}, \ldots, h_{m}$ in $F_{n}(J)$.

The center of $F_{m}(J)$ is a commutative domain and its transcendence degree is $d=(m-1) k^{2}+1$ (see for example [8, p. 105]). Let

$$
c_{1}=c_{1}\left(y_{1}, \ldots, y_{m}\right), \ldots, c_{d}=c_{d}\left(y_{1}, \ldots, y_{m}\right)
$$

be a transcendence basis of the center of $F_{m}(J)$ (so the $c_{i}\left(x_{1}, \ldots, x_{m}\right)$ are central polynomials for the $k \times k$ matrix algebra). The algebraic independence of $h_{1}, \ldots, h_{m}$ in $F_{n}(J)$ together with the algebraic independence (in the ordinary sense) of $c_{1}, \ldots, c_{d}$ in the center of $F_{m}(J)$ implies that $c_{i}\left(h_{1}, \ldots, h_{m}\right)(i=1, \ldots, d)$ are algebraically independent in the center of $F_{n}(J)$. But this center has transcendence degree $(n-1) k^{2}+1$, implying that $m \leq n$.

We use the following crucial argument from [8] or [9]. Any non-zero T-ideal $I$ is contained in the T-ideal generated by $\left[x_{1}, x_{2}\right]$. So we have the natural onto homomorphism

$$
F_{n}(I) \rightarrow K\left[x_{1}, \ldots, x_{n}\right]
$$

which commutes with the action of $G$, and by the complete reducibility of this action the homomorphism

$$
F_{n}(I)^{G} \rightarrow K\left[x_{1}, \ldots, x_{n}\right]^{G}
$$

is also onto. Therefore the image of a generating set of $F_{n}(I)^{G}$ is a generating set of $K\left[x_{1}, \ldots, x_{n}\right]^{G}$, hence $F_{n}(I)^{G}$ can not be generated by less than $n$ elements. Thus if $F_{n}(I)^{G}$ is generated by algebraically independent elements, then by Proposition 2.1 it is generated by $n$ elements.

Assume that $f_{1}, \ldots, f_{n}$ generate $F_{n}(I)^{G}$. Their images in $K\left[x_{1}, \ldots, x_{n}\right]$ generate $K\left[x_{1}, \ldots, x_{n}\right]^{G}$, hence $G$ must be a pseudo-reflection group. We may assume by $[9$, Lemma 2] that $f_{1}, \ldots, f_{n}$ are homogeneous, therefore their degrees are the degrees of $G$. Moreover, the proof of [9, Lemma 2] shows that if $h_{1}, \ldots, h_{n} \in F_{n}(I)^{G}$ such that their images generate $K\left[x_{1}, \ldots, x_{n}\right]^{G}$, then $F_{n}(I)^{G}=K\left\langle h_{1}, \ldots, h_{n}\right\rangle$.

By the above discussion Theorem 1.2 splits into the next two statements: 
Theorem 2.2. Let $G$ be a pseudo-reflection group and let $I$ be a T-ideal containing $\left[\left[x_{2}, x_{1}\right], x_{1}\right]$. If $f_{1}, \ldots, f_{n}$ are homogeneous elements in $F_{n}(I)^{G}$ such that their images generate $K\left[x_{1}, \ldots, x_{n}\right]^{G}$, then $f_{1}, \ldots, f_{n}$ are algebraically independent in $F_{n}(I)$ and they generate $F_{n}(I)^{G}$. In particular, $F_{n}(I)^{G} \cong F_{n}(I)$.

Theorem 2.3. If the T-ideal I does not contain the polynomial $\left[\left[x_{2}, x_{1}\right], x_{1}\right]$, then $F_{n}(I)^{G}$ can not be generated by $n$ elements for any $n \geq 2$ and finite group $G<$ $G l_{n}(K)$.

We recall some basic facts about the T-ideals of the unitary free associative $K$-algebra. Let $B$ denote the subalgebra of $K\left\langle x_{1}, x_{2}, \ldots\right\rangle$ generated by all the commutators $\left[x_{i_{1}}, \ldots, x_{i_{r}}\right]$ with $r \geq 2$, where $\left[x_{1}, \ldots, x_{r}\right]$ is defined inductively by $\left[x_{1}, \ldots, x_{r}\right]=\left[\left[x_{1}, \ldots, x_{r-1}\right], x_{r}\right]$ for $r \geq 3$. The elements of $B$ are called proper polynomials, and they can be characterised as the polynomials with zero partial derivatives. Clearly, $B_{n}=B \cap K\left\langle x_{1}, \ldots, x_{n}\right\rangle$ is a $G l_{n}$-submodule of $K\left\langle x_{1}, \ldots, x_{n}\right\rangle$. Drensky showed (see [5, Theorem 2.2]) that any T-ideal $I$ of the free unitary algebra is generated by the proper polynomials that it contains, and as $G l_{n}$-modules

$$
F_{n}(I) \cong K\left[x_{1}, \ldots, x_{n}\right] \otimes\left(B_{n} / B_{n} \cap I\right) .
$$

Both $I_{n}$ and $B_{n}$ are multigraded subalgebras with respect to the usual multigrading on $K\left\langle x_{1}, \ldots, x_{n}\right\rangle$, so $F_{n}(I)$ and $B_{n} / B_{n} \cap I$ inherit the multigrading.

For any $\alpha=\left(\alpha_{1}, \ldots, \alpha_{n}\right)$ and multigraded algebra $R$ we put

$$
R^{(\alpha)}=\{f \in R \mid f \text { is multihomogeneous of multidegree } \alpha\},
$$

and for any $\mathbb{N}$-graded algebra $R$ and non-negative integer $d$

$$
R^{(d)}=\{f \in R \mid f \text { is homogeneous of total degree } d\} .
$$

Obviously $B_{n}^{(0)}=K$ and $B_{n}^{(1)}=\emptyset$. If $n \geq 2$, then $B_{n}^{(2)}$ is an irreducible $G l_{n}$-module generated by $\left[x_{1}, x_{2}\right]$, and $B_{n}^{(3)}$ is an irreducible $G l_{n}-$ module generated by $\left[x_{2}, x_{1}, x_{1}\right]$. Therefore there exists a unique maximal T-ideal $M$ among the T-ideals not containing $\left[x_{2}, x_{1}, x_{1}\right], M$ is generated by the proper polynomials of degree greater than 3 (in particular, $M$ has no elements of degree less than 4). If $I$ is a T-ideal not containing $\left[x_{2}, x_{1}, x_{1}\right]$, then $F_{n}(M)^{G}$ is a homomorphic image of $F_{n}(I)^{G}$, thus Theorem 2.3 is a consequence of the following more special statement:

Proposition 2.4. Let $M$ be the T-ideal generated by the proper polynomials of degree greater than 3 , and let $G<G l_{n}(n \geq 2)$ be a finite pseudo-reflection group. Then $F_{n}(M)^{G}$ can not be generated by $n$ elements.

The Hilbert series of a multigraded algebra $R$ is an $n$ variable formal power series defined by

$$
H\left(R ; t_{1}, \ldots, t_{n}\right)=\sum_{\alpha=\left(\alpha_{1}, \ldots, \alpha_{n}\right)} \operatorname{dim}_{K}\left(R^{(\alpha)}\right) t_{1}^{\alpha_{1}} \ldots t_{n}^{\alpha_{n}},
$$

and the Hilbert series of an $\mathbb{N}$-graded algebra $R$ is the formal power series

$$
H(R ; t)=\sum_{d=0}^{\infty} \operatorname{dim}_{K}\left(R^{(d)}\right) t^{d}
$$


Let $G$ be a finite subgroup of $G l_{n}(K)$. For any element $g \in G$ denote by $\omega_{1}(g), \ldots$, $\omega_{n}(d)$ the eigenvalues of $g$ in the algebraic closure of $K . F_{n}(I)^{G}$ is an $\mathbb{N}$-graded algebra, and we have the noncommutative Molien-Weyl formula (see [8, Theorem 7]):

$$
H\left(F_{n}(I)^{G} ; t\right)=\frac{1}{|G|} \sum_{g \in G} H\left(F_{n}(I) ; \omega_{1}(g) t, \ldots, \omega_{n}(g) t\right)
$$

\section{Proof of Theorem 2.2}

Denote by $J$ the T-ideal generated by $\left[x_{2}, x_{1}, x_{1}\right]$, and let

$$
s_{d}\left(x_{1}, \ldots, x_{d}\right)=\sum_{\pi \in \operatorname{Sym}(d)}(-1)^{\pi} x_{\pi(1)} \ldots x_{\pi(d)}
$$

be the standard polynomial. It is well known (see [6, 3.2.1. Theorem]) that $B_{n}^{(d)} / B_{n}^{(d)} \cap J=0$, if $d$ is odd or $d>n$, and it is an irreducible $G l_{n}$-module generated by $s_{d}\left(y_{1}, \ldots, y_{d}\right)$ if $d$ is even and $d \leq n$. Let $J(m)$ be the T-ideal generated by $\left[x_{2}, x_{1}, x_{1}\right]$ and $s_{2 m}\left(x_{1}, \ldots, x_{2 m}\right)$, so the only T-ideals containing $\left[x_{2}, x_{1}, x_{1}\right]$ are $J$ and $J(m)(m=1,2, \ldots)$. We note that

$$
K\left\langle x_{1}, \ldots, x_{n}\right\rangle / J(m)_{n}=K\left\langle x_{1}, \ldots, x_{n}\right\rangle / J_{n} \quad \text { if } 2 m>n .
$$

Let $f_{1}, \ldots, f_{n} \in F_{n}(J(m))^{G}$ be homogeneous invariants such that their images in $K\left[x_{1}, \ldots, x_{n}\right]$ generate $K\left[x_{1}, \ldots, x_{n}\right]^{G}$. We prove by induction on $m$ that $f_{1}, \ldots, f_{n}$ are algebraically independent in $F_{n}(J(m))$. In the case $m=1$ there is nothing to prove. Suppose that the statement is true for $F_{n}(J(k))$, where $k \leq m$. We may assume that $2 m \leq n$. The algebra $F_{n}(J(m+1))$ has a linear basis (see [6])

$$
\left\{y_{1}^{\alpha_{1}} \ldots y_{n}^{\alpha_{n}} s_{2 k}\left(y_{i_{1}}, \ldots, y_{i_{2 k}}\right) \mid \alpha_{1}, \ldots, \alpha_{n} \in \mathbb{N}, 0 \leq k \leq m, 1 \leq i_{1}<\ldots<i_{2 k} \leq n\right\} \text {. }
$$

Suppose that $h\left(f_{1}, \ldots, f_{n}\right)=0$ in $F_{n}(J(m+1))$ for some $h \in K\left\langle x_{1}, \ldots, x_{n}\right\rangle$ with $h \notin J(m+1)_{n}$. Then we may assume that $h$ is of the form

$$
h=\sum_{\alpha=\left(\alpha_{1}, \ldots, \alpha_{n}\right)} \sum_{k=0}^{m} \sum_{1 \leq i_{1}<\ldots<i_{2 k} \leq n} a_{\alpha, k, i} x_{1}^{\alpha_{1}} \ldots x_{n}^{\alpha_{n}} s_{2 k}\left(x_{i_{1}}, \ldots, x_{i_{2 k}}\right),
$$

where at least one of the coefficients $a_{\alpha, k, i}$ is non-zero. Let $k_{0}$ be the minimal $k$ such that $a_{\alpha, i, k} \neq 0$ for some $\alpha, i$. If $k_{0} \leq m-1$, then by the induction hypothesis

$$
\sum_{\alpha, i} a_{\alpha, k_{0}, i} f_{1}^{\alpha_{1}} \ldots f_{n}^{\alpha_{n}} s_{2 k_{0}}\left(f_{i_{1}}, \ldots, f_{i_{2 k_{0}}}\right) \notin J_{n}\left(k_{0}+1\right) / J_{n}(m+1) .
$$

On the other hand

$$
\left.\sum_{\alpha, k>k_{0}, i} a_{\alpha, k, i} f_{1}^{\alpha_{1}} \ldots f_{n}^{\alpha_{n}} s_{2 k}\left(f_{i_{1}}, \ldots, f_{i_{2 k}}\right) \equiv 0 \text { (modulo } J_{n}\left(k_{0}+1\right) / J_{n}(m+1)\right),
$$


contradicting that $h\left(f_{1}, \ldots, f_{n}\right)=0$ in $F_{n}(J(m+1))$. Thus $k_{0}=m$ and we have

$$
\sum_{\alpha, i} a_{\alpha, i} f_{1}^{\alpha_{1}} \ldots f_{n}^{\alpha_{n}} s_{2 m}\left(f_{i_{1}}, \ldots, f_{i_{2 m}}\right)=0
$$

in $F_{n}(J(m+1))$.

Define the maps

$$
\frac{\partial}{\partial y_{i}}: F_{n}(J(m+1)) \rightarrow F_{n}(J(m+1)) \quad(i=1, \ldots, n)
$$

in the following way. If $f=f\left(y_{1}, \ldots, y_{n}\right) \in F_{n}(J(m+1))$ is multihomogeneous of multidegree $\alpha=\left(\alpha_{1}, \ldots, \alpha_{n}\right)$, then let $\frac{\partial}{\partial y_{i}} f$ be the multihomogeneous component of multidegree $\left(\alpha_{1}, \ldots, \alpha_{i}-1, \ldots, \alpha_{n}\right)$ of $f\left(y_{1}+1, \ldots, y_{n}+1\right)\left(\frac{\partial}{\partial y_{i}} f=0\right.$ if $\left.\alpha_{i}=0\right)$. Now extend $\frac{\partial}{\partial y_{i}}$ to $F_{n}(J(m+1))$ by linearity. Clearly, $\frac{\partial}{\partial y_{i}}$ is a derivation.

Lemma 3.1. For any $f, g \in F_{n}(J(m+1))$ we have the equality

$$
[f, g] \equiv \sum_{1 \leq i, j \leq n} \frac{\partial f}{\partial y_{i}} \frac{\partial g}{\partial y_{j}}\left[y_{i}, y_{j}\right] \quad\left(\text { modulo } C^{2}\right)
$$

where

$$
C=F_{n}(J(m+1))\left[F_{n}(J(m+1)), F_{n}(J(m+1))\right] F_{n}(J(m+1))
$$

is the commutator ideal of $F_{n}(J(m+1))$.

Proof. By the multilinearity of the derivations and [, ] it suffices to prove the lemma when

$$
f=y_{i_{1}} \ldots y_{i_{k}} \text { and } g=y_{j_{1}} \ldots y_{j_{l}}
$$

are monomials. We have

$$
\begin{aligned}
& \left(y_{i_{1}} \ldots y_{i_{k}}\right)\left(y_{j_{1}} \ldots y_{j_{l}}\right) \\
& =y_{i_{1}} \ldots y_{i_{k-1}} y_{j_{1}} y_{i_{k}} y_{j_{2}} \ldots y_{j_{l}}+y_{i_{1}} \ldots y_{i_{k-1}}\left[y_{i_{k}}, y_{j_{1}}\right] y_{j_{2}} \ldots y_{j_{l}} \\
& \quad=\left(y_{i_{1}} \ldots y_{i_{k-1}}\right)\left(y_{j_{2}} \ldots y_{j_{l}}\right)\left[y_{i_{k}}, y_{j_{1}}\right]+y_{i_{1}} \ldots y_{i_{k-1}} y_{j_{1}} y_{i_{k}} y_{j_{2}} \ldots y_{j_{l}}
\end{aligned}
$$

(the second equality follows from the fact that any commutator lies in the center of $\left.F_{n}(J(m+1))\right)$. Now exchange $y_{i_{k-1}}$ and $y_{j_{1}}$ in the second term of the above sum, that is, replace this term by

$$
y_{i_{1}} \ldots y_{i_{k-2}} y_{j_{1}} y_{i_{k-1}} y_{i_{k}} y_{j_{2}} \ldots y_{j_{l}}+\left(y_{i_{1}} \ldots y_{i_{k-2}} y_{j_{k}}\right)\left(y_{j_{2}} \ldots y_{j_{l}}\right)\left[y_{i_{k-1}}, y_{j_{1}}\right] \text {. }
$$

Continuing this process we obtain

$$
\begin{aligned}
& \left(y_{i_{1}} \ldots y_{i_{k}}\right)\left(y_{j_{1}} \ldots y_{j_{l}}\right) \\
& \quad=y_{j_{1}}\left(y_{i_{1}} \ldots y_{i_{k}}\right)\left(y_{j_{2}} \ldots y_{j_{l}}\right)+\sum_{r=1}^{k}\left(y_{i_{1}} \ldots \hat{y}_{i_{r}} \ldots y_{i_{k}}\right)\left(y_{j_{2}} \ldots y_{j_{l}}\right)\left[y_{i_{r}}, y_{j_{1}}\right]
\end{aligned}
$$


where the sign $\hat{y}_{i_{r}}$ means that we delete $y_{i_{r}}$ in the word $y_{i_{1}} \ldots y_{i_{k}}$. On applying the same process to the word $\left(y_{i_{1}} \ldots y_{i_{k}}\right)\left(y_{j_{2}} \ldots y_{j_{l}}\right)$ we obtain that

$$
\begin{aligned}
&\left(y_{i_{1}} \ldots y_{i_{k}}\right)\left(y_{j_{1}} \ldots y_{j_{l}}\right)= \sum_{r=1}^{k}\left(y_{i_{1}} \ldots \hat{y}_{i_{r}} \ldots y_{i_{k}}\right)\left(y_{j_{2}} \ldots y_{j_{l}}\right)\left[y_{i_{r}}, y_{j_{1}}\right] \\
&+ \sum_{r=1}^{k} y_{j_{1}}\left(y_{i_{1}} \ldots \hat{y}_{i_{r}} \ldots y_{i_{k}}\right)\left(y_{j_{3}} \ldots y_{j_{l}}\right)\left[y_{i_{r}}, y_{j_{2}}\right] \\
&+y_{j_{1}} y_{j_{2}}\left(y_{i_{1}} \ldots y_{i_{k}}\right)\left(y_{j_{3}} \ldots y_{j_{l}}\right) \\
& \stackrel{\left(\bmod _{\equiv} C^{2}\right)}{\equiv} \sum_{r=1}^{k}\left(y_{i_{1}} \ldots \hat{y}_{i_{r}} \ldots y_{i_{k}}\right)\left(\hat{y}_{j_{1}} y_{j_{2}} \ldots y_{j_{l}}\right)\left[y_{i_{r}}, y_{j_{1}}\right] \\
&+\sum_{r=1}^{k}\left(y_{i_{1}} \ldots \hat{y}_{i_{r}} \ldots y_{i_{k}}\right)\left(y_{j_{1}} \hat{y}_{j_{2}} y_{j_{3}} \ldots y_{j_{l}}\right)\left[y_{i_{r}}, y_{j_{2}}\right] \\
& \quad+y_{j_{1}} y_{j_{2}}\left(y_{i_{1}} \ldots y_{i_{k}}\right)\left(y_{j_{3}} \ldots y_{j_{l}}\right) \\
&=\sum_{i=1}^{n}\left(\frac{\partial}{\partial y_{i}}\left(y_{i_{1}} \ldots y_{i_{k}}\right)\right)\left(\hat{y}_{j_{1}} y_{j_{2}} \ldots y_{j_{l}}\right)\left[y_{i}, y_{j_{1}}\right] \\
&+\sum_{i=1}^{n}\left(\frac{\partial}{\partial y_{i}}\left(y_{i_{1}} \ldots y_{i_{k}}\right)\right)\left(y_{j_{1}} \hat{y}_{j_{2}} \ldots y_{j_{l}}\right)\left[y_{i}, y_{j_{2}}\right] \\
& \quad+y_{j_{1}} y_{j_{2}}\left(y_{i_{1}} \ldots y_{i_{k}}\right)\left(y_{j_{3}} \ldots y_{j_{l}}\right) .
\end{aligned}
$$

Repeating this algorithm finally we get

$$
\begin{aligned}
& \left(y_{i_{1}} \ldots y_{i_{k}}\right)\left(y_{j_{1}} \ldots y_{j_{l}}\right) \\
& \left.\stackrel{(\bmod }{\equiv} C^{2}\right)\left(y_{j_{1}} \ldots y_{j_{l}}\right)\left(y_{i_{1}} \ldots y_{i_{k}}\right)+\sum_{i=1}^{n} \sum_{r=1}^{l}\left(\frac{\partial}{\partial y_{i}}\left(y_{i_{1}} \ldots y_{i_{k}}\right)\right)\left(y_{j_{1}} \ldots \hat{y}_{j_{r}} \ldots y_{j_{l}}\right)\left[y_{i}, y_{j_{r}}\right] \\
& \quad=\left(y_{j_{1}} \ldots y_{j_{l}}\right)\left(y_{i_{1}} \ldots y_{i_{k}}\right)+\sum_{i=1}^{n} \sum_{j=1}^{n}\left(\frac{\partial}{\partial y_{i}}\left(y_{i_{1}} \ldots y_{i_{k}}\right)\right)\left(\frac{\partial}{\partial y_{j}}\left(y_{j_{1}} \ldots y_{j_{l}}\right)\right)\left[y_{i}, y_{j}\right]
\end{aligned}
$$

which explicitly shows the claim.

Now we use Lemma 3.1 to rewrite $A=s_{2 m}\left(f_{1}, \ldots, f_{2 m}\right)$. By the definition of $s_{2 m}$

$$
A=\frac{1}{2^{m}} \sum_{\pi \in \operatorname{Sym}(2 m)}(-1)^{\pi}\left[f_{\pi(1)}, f_{\pi(2)}\right] \ldots\left[f_{\pi(2 m-1)}, f_{\pi(2 m)}\right] .
$$

We have $C^{m+1}=0$ in $F_{n}(J(m+1))$, because the commutators are central elements of $F_{n}(J(m+1))$ and any proper polynomial of degree greater than $2 m+1$ is contained in $J(m+1)$. Therefore in the right hand side of the above equality we may replace $\left[f_{\pi(2 k-1)}, f_{\pi(2 k)}\right]$ by something that is congruent with it modulo $C^{2}$. 
So by Lemma 3.1 we have

$$
\begin{aligned}
A= & \frac{1}{2^{m}} \sum_{\pi \in S y m(2 m)}(-1)^{\pi} \sum_{\left(i_{1}, \ldots, i_{2 m}\right)} \frac{\partial f_{\pi(1)}}{\partial y_{i_{1}}} \frac{\partial f_{\pi(2)}}{\partial y_{i_{2}}}\left[y_{i_{1}}, y_{i_{2}}\right] \\
& \ldots \frac{\partial f_{\pi(2 m-1)}}{\partial y_{i_{2 m-1}}} \frac{\partial f_{\pi(2 m)}}{\partial y_{i_{2 m}}}\left[y_{i_{2 m-1}}, y_{i_{2 m}}\right] \\
= & \frac{1}{2^{m}} \sum_{\pi \in \operatorname{Sym}(2 m)}(-1)^{\pi} \sum_{\left(i_{1}, \ldots, i_{2 m}\right)} \frac{\partial f_{\pi(1)}}{\partial y_{i_{1}}} \ldots \frac{\partial f_{\pi(2 m)}}{\partial y_{i_{2 m}}}\left[y_{i_{1}}, y_{i_{2}}\right] \ldots\left[y_{i_{2 m-1}}, y_{i_{2 m}}\right] .
\end{aligned}
$$

The polynomial $\left[x_{1}, x_{2}\right]\left[x_{1}, x_{3}\right]$ is contained in $J$, hence if the $i_{1}, \ldots, i_{2 m}$ are not pairwise different, then the corresponding term is zero in the above expression, implying that

$$
\begin{aligned}
& A= \frac{1}{2^{m}} \sum_{\pi \in S y m(2 m)}(-1)^{\pi} \sum_{1 \leq i_{1}<\ldots<i_{2 m} \leq n} \sum_{\rho \in S y m(2 m)} \frac{\partial f_{\pi(1)}}{\partial y_{i_{\rho(1)}}} \\
& \cdots \frac{\partial f_{\pi(2 m)}}{\partial y_{i_{\rho(2 m)}}}\left[y_{i_{\rho(1)}}, y_{i_{\rho(2)}}\right] \ldots\left[y_{i_{\rho(2 m-1)}}, y_{i_{\rho(2 m)}}\right] \\
&= \sum_{1 \leq i_{1}<\ldots<i_{2 m} \leq n} \frac{1}{2^{m}} \sum_{\rho \in S y m(2 m)}(-1)^{\rho}\left[y_{i_{\rho(1)}}, y_{i_{\rho(2)}}\right] \ldots\left[y_{i_{\rho(2 m-1)}}, y_{i_{\rho(2 m)}}\right] \\
& \times \sum_{\pi \in \operatorname{Sym}(2 m)}(-1)^{\pi}(-1)^{\rho} \frac{\partial f_{\pi(1)}}{\partial y_{i_{\rho(1)}}} \ldots \frac{\partial f_{\pi(2 m)}}{\partial y_{i_{\rho(2 m)}}} .
\end{aligned}
$$

Again by $C^{m+1}=0$ we may permute $\frac{\partial f_{\pi(1)}}{\partial y_{i_{\rho(1)}}}, \ldots, \frac{\partial f_{\pi(2 m)}}{\partial y_{i_{\rho(2 m)}}}$ among each other in the above expression, and we get

$$
\left.A=\sum_{1 \leq i_{1}<\ldots<i_{2 m} \leq n} \sum_{\sigma \in \operatorname{Sym}(2 m)}(-1)^{\sigma} \frac{\partial f_{1}}{\partial y_{i_{\sigma(1)}}} \ldots \frac{\partial f_{2 m}}{\partial y_{i_{\sigma(2 m)}}}\right) s_{2 m}\left(y_{i_{1}}, \ldots, y_{i_{2 m}}\right) .
$$

For any $1 \leq i_{1}<\ldots<i_{2 m} \leq n$ and $1 \leq j_{1}<\ldots<j_{2 m} \leq n$ we put

$$
f_{j_{1}, \ldots, j_{2 m}}^{i_{1}, \ldots, i_{2 m}}=\sum_{\sigma \in \operatorname{Sym}(2 m)}(-1)^{\sigma} \frac{\partial f_{i_{1}}}{\partial y_{j_{\sigma(1)}}} \ldots \frac{\partial f_{i_{2 m}}}{\partial y_{j_{\sigma(2 m)}}} .
$$

Consider the natural homomorphism

$$
\psi: F_{n}(J(m+1)) \rightarrow K\left[x_{1}, \ldots, x_{n}\right] .
$$

The image of any $f \in F_{n}(J(m+1))$ has a normal form

$$
\psi(f)=\sum_{\alpha=\left(\alpha_{1}, \ldots, \alpha_{n}\right)} b_{\alpha} x_{1}^{\alpha_{1}} \ldots x_{n}^{\alpha_{n}} .
$$

Now let $\phi$ be the map $F_{n}(J(m+1)) \rightarrow F_{n}(J(m+1))$ defined by

$$
\phi(f)=\sum_{\alpha} b_{\alpha} y_{1}^{\alpha_{1}} \ldots y_{n}^{\alpha_{n}} .
$$


Obviously, we have $\phi(f) \equiv f(\bmod C)$, hence $C^{m+1}=0$ implies that

$$
f s_{2 m}\left(y_{i_{1}}, \ldots, y_{i_{2 m}}\right)=\phi(f) s_{2 m}\left(y_{i_{1}}, \ldots, y_{i_{2 m}}\right) .
$$

Summarizing, we have

$$
\begin{aligned}
& 0=h\left(f_{1}, \ldots, f_{n}\right) \sum_{\alpha=\left(\alpha_{1}, \ldots, \alpha_{n}\right)} \sum_{1 \leq i_{1}<\ldots<i_{2 m} \leq n} a_{\alpha, i} f_{1}^{\alpha_{1}} \ldots f_{n}^{\alpha_{n}} s_{2 m}\left(f_{i_{1}}, \ldots, f_{i_{2 m}}\right) \\
& \quad=\sum_{1 \leq j_{1}<\ldots<j_{2 m} \leq n} \phi\left(\sum_{\alpha, i} a_{\alpha, i} f_{1}^{\alpha_{1}} \ldots f_{n}^{\alpha_{n}} f_{j_{1}, \ldots, j_{2 m}}^{i_{1}, \ldots, i_{2 m}}\right) s_{2 m}\left(y_{j_{1}}, \ldots, y_{j_{2 m}}\right) .
\end{aligned}
$$

Now since the elements $y_{1}^{\alpha_{1}} \ldots y_{n}^{\alpha_{n}} s_{2 m}\left(y_{i_{1}}, \ldots, y_{i_{2 m}}\right)$ are linearly independent in $F_{n}(J(m+1))$,

$$
\phi\left(\sum_{\alpha, i} a_{\alpha, i} f_{1}^{\alpha_{1}} \ldots f_{n}^{\alpha_{n}} f_{j_{1}, \ldots, j_{2 m}}^{i_{1}, \ldots, i_{2 m}}\right)
$$

must be zero for all $j_{1}, \ldots, j_{2 m}$, implying that

$$
\psi\left(\sum_{\alpha, i} a_{\alpha, i} f_{1}^{\alpha_{1}} \ldots f_{n}^{\alpha_{n}} f_{j_{1}, \ldots, j_{2 m}}^{i_{1}, \ldots, i_{2 m}}\right)=0
$$

because the monomials $y_{1}^{\beta_{1}} \ldots y_{n}^{\beta_{n}}$ are linearly independent in $F_{n}(J(m+1))$.

Introduce the symbols $d x_{1}, \ldots, d x_{n}$, and let

$$
E=K\left\langle d x_{1}, \ldots, d x_{n} \mid d x_{i} d x_{j}=-d x_{j} d x_{i}, 1 \leq i, j \leq n\right\rangle
$$

be the Grassmann algebra of an $n$ dimensional linear space. Consider the map

$$
d: K\left[x_{1}, \ldots, x_{n}\right] \rightarrow K\left[x_{1}, \ldots, x_{n}\right] \otimes E
$$

defined by

$$
d f=d(f)=\sum_{i=1}^{n} \frac{\partial f}{\partial x_{i}} d x_{i} .
$$

The commutative polynomials $h_{i}=\psi\left(f_{i}\right)(i=1, \ldots, n)$ form an algebraically independent generating set of $K\left[x_{1}, \ldots, x_{n}\right]^{G}$. Direct computation shows that

$$
\begin{array}{rl}
\sum_{\alpha, i} a_{\alpha, i} h_{1}^{\alpha_{1}} \ldots h_{n}^{\alpha_{n}} & d h_{i_{1}} \ldots d h_{i_{2 m}} \\
& =\sum_{1 \leq j_{1}<\ldots<j_{2 m} \leq n} \psi\left(\sum_{\alpha, i} a_{\alpha, i} f_{1}^{\alpha_{1}} \ldots f_{n}^{\alpha_{n}} f_{j_{1}, \ldots, j_{2 m}}^{i_{1}, \ldots, i_{2 m}}\right) d x_{j_{1}} \ldots d x_{j_{n}}
\end{array}
$$

and by our hypothesis the latter is zero. Now [14, Theorem] implies that

$$
\left\{h_{1}^{\alpha_{1}} \ldots h_{n}^{\alpha_{n}} d h_{i_{1}} \ldots d h_{i_{2 m}} \mid \alpha_{1}, \ldots, \alpha_{n} \in \mathbb{N}, 1 \leq i_{1}<\ldots<i_{2 m} \leq n\right\}
$$

is a linearly independent subset of $K\left[x_{1}, \ldots, x_{n}\right] \otimes E$, hence $a_{\alpha, i}=0$ for every $\alpha, i$, contradicting the hypothesis that $f_{1}, \ldots, f_{n} \in F_{n}(J(m+1))$ are algebraically dependent. 
Remark. The above argument did not use the fact that $f_{1}, \ldots, f_{n}$ are invariants. So we have proved for all $m=1,2, \ldots$ that if $f_{1}, \ldots, f_{n} \in F_{n}(J(m))$ are algebraically independent (in the ordinary sense) modulo the commutator ideal, then they are algebraically independent in $F_{n}(J(m))$.

We have to show that $f_{1}, \ldots, f_{n}$ generate $F_{n}(J(m))^{G}$. They are homogeneous of degree $d_{1}, \ldots, d_{n}$, and as we pointed out earlier these are the degrees of $G$. We conclude from the formula (2.1) and the $G l_{n}$-structure of $B_{n} / B_{n} \cap J(m)$ that the Hilbert series of $F_{n}(J(m))$ is

$$
H\left(F_{n}(J(m)) ; t_{1}, \ldots, t_{n}\right)=\frac{\sum_{i=0}^{m-1} \sigma_{2 i}\left(t_{1}, \ldots, t_{n}\right)}{\prod_{j=1}^{n}\left(1-t_{j}\right)},
$$

where $\sigma_{k}$ is the $k$ th elementary symmetric function $\left(\sigma_{0}=1\right.$ and $\sigma_{k}=0$ if $\left.k>n\right)$. We have the isomorphism

$$
K\left\langle f_{1}, \ldots, f_{n}\right\rangle \cong F_{n}(J(m)),
$$

hence the $\mathbb{N}$-graded Hilbert series of the graded subalgebra $K\left\langle f_{1}, \ldots, f_{n}\right\rangle$ of $F_{n}(J(m))^{G}$ is

$$
H\left(F_{n}(J(m)) ; t^{d_{1}}, \ldots, t^{d_{n}}\right)=\frac{\sum_{i=0}^{m-1} \sigma_{2 i}\left(t^{d_{1}}, \ldots, t^{d_{n}}\right)}{\prod_{j=1}^{n}\left(1-t^{d_{j}}\right)}
$$

Using the noncommutative Molien-Weyl formula (2.2)

$$
H\left(F_{n}(J(m))^{G} ; t\right)=\frac{1}{|G|} \sum_{g \in G} \frac{\sum_{i=0}^{m-1} \sigma_{2 i}\left(\omega_{1}(g) t, \ldots, \omega_{n}(g) t\right)}{\prod_{j=1}^{n}\left(1-\omega_{j}(g) t\right)} .
$$

Solomon's formula

$$
\frac{1}{|G|} \sum_{g \in G} \frac{\sigma_{p}\left(\omega_{1}(g), \ldots, \omega_{n}(g)\right)}{\prod_{j=1}^{n}\left(1-\omega_{j}(g) t\right)}=\frac{\sigma_{p}\left(t^{d_{1}-1}, \ldots, t^{d_{n}-1}\right)}{\prod_{j=1}^{n}\left(1-t^{d_{j}}\right)}
$$

(see [14, formula (5)]) says that $F_{n}(J(m))^{G}$ has the same Hilbert series as its subalgebra $K\left\langle f_{1}, \ldots, f_{n}\right\rangle$, therefore the two algebras must coincide. This finishes the proof of Theorem 2.2.

Remark. The polynomial $\left[x_{2}, x_{1}, x_{1}\right]$ appears in the theory of PI-algebras as a generator of the T-ideal of identities of the infinite dimensional Grassmann algebra (cf. [11]). It is interesting to note that both parts of the above proof use Solomon's results from [14] on a pseudo-reflection group acting on the tensor product of $K\left[x_{1}, \ldots, x_{n}\right]$ and the Grassmann algebra of an $n$ dimensional vector space.

\section{Proof of Proposition 2.4}

Let $G$ be a pseudo-reflection group, and suppose that $F_{n}(M)^{G}$ is generated by $n$ elements (where $M$ is the T-ideal generated by all the proper polynomials of degree greater than 3). As we mentioned in Section 2, then $F_{n}(M)^{G}$ is generated by its homogeneous elements $f_{1}, \ldots, f_{n}$ if and only if their images in $K\left[x_{1}, \ldots, x_{n}\right]$ 
generate $K\left[x_{1}, \ldots, x_{n}\right]^{G}$. In the sequel we assume that $f_{1}, \ldots, f_{n} \in F_{n}(M)^{G}$ have this property. By $(2.1)$ the Hilbert series of $F_{n}(M)$ is

$$
H\left(F_{n}(M) ; t_{1}, \ldots, t_{n}\right)=\frac{1+S_{(1,1)}\left(t_{1}, \ldots, t_{n}\right)+S_{(2,1)}\left(t_{1}, \ldots, t_{n}\right)}{\prod_{i=1}^{n}\left(1-t_{i}\right)},
$$

where $S_{\lambda}\left(t_{1}, \ldots, t_{n}\right)$ is the Schur function corresponding to the partition $\lambda=$ $\left(\lambda_{1}, \ldots, \lambda_{n}\right)$. On expressing the Schur functions $S_{(1,1)}$ and $S_{(2,1)}$ by the elementary symmetric polynomials we obtain

$$
H\left(F_{n}(M) ; t_{1}, \ldots, t_{n}\right)=\frac{1+\sigma_{2}\left(t_{1}, \ldots, t_{n}\right)-\sigma_{3}\left(t_{1}, \ldots, t_{n}\right)+\sigma_{1} \sigma_{2}\left(t_{1}, \ldots, t_{n}\right)}{\prod_{i=1}^{n}\left(1-t^{d_{i}}\right)} .
$$

Since $F_{n}(M)^{G}$ is generated by $f_{1}, \ldots, f_{n}$, it is an $\mathbb{N}$-graded homomorphic image of $F_{n}(M)$, where we give the degrees $d_{1}, \ldots, d_{n}$ to the generators of $F_{n}(M)$. Hence in the formal power series

$$
D(t)=H\left(F_{n}(M) ; t^{d_{1}}, \ldots, t^{d_{n}}\right)-H\left(F_{n}(M)^{G} ; t\right)
$$

each coefficient is a non-negative integer. By (2.2) and (3.1) we have

$$
D(t)=\frac{F(t)}{\prod_{i=1}^{n}\left(1-t^{d_{i}}\right)}-H_{1}(t),
$$

where

$$
F(t)=\left(t^{d_{1}}+\ldots+t^{d_{n}}\right) \sum_{1 \leq i<j \leq n} t^{d_{i}+d_{j}}
$$

and

$$
H_{1}(t)=\frac{1}{|G|} \sum_{g \in G} \frac{\left(\omega_{1}(g) t+\ldots+\omega_{n}(g) t\right)\left(\sum_{1 \leq i<j \leq n} \omega_{i}(g) \omega_{j}(g) t^{2}\right)}{\prod_{i=1}^{n}\left(1-\omega_{i}(g) t\right)} .
$$

Steinberg proved (see [1, p. 127]) that

$$
\sum_{1 \leq i<j \leq n} \omega_{i} \omega_{j}: G \rightarrow \mathbb{C}
$$

is an irreducible character of $G$. (Actually, $\sum \omega_{i} \omega_{j}$ is the character of $G<G l(V)$ acting on the second exterior power of $V$, and we do not need here the irreducibility.) Therefore

$$
\left(\bar{\omega}_{1}+\ldots+\bar{\omega}_{n}\right) \sum_{1 \leq i<j \leq n} \bar{\omega}_{i} \bar{\omega}_{j}
$$

is a character of degree $n\left(\begin{array}{l}n \\ 2\end{array}\right)$ of $G$, and we may decompose it as $\sum_{i=1}^{r} m_{i} \chi_{i}$, where $\chi_{1}, \ldots, \chi_{r}$ are pairwise different irreducible characters of $G$ and $m_{1}, \ldots, m_{r}$ are positive integers. Let $I$ be the ideal of $K\left[x_{1}, \ldots, x_{n}\right]$ generated by the invariants of $G$ of strictly positive degree. Chevalley showed in [2] that the representation of 
$G$ on $K\left[x_{1}, \ldots, x_{n}\right] / I$ is equivalent to the regular representation. Following [15] we associate with any irreducible character $\chi$ of $G$ the polynomial

$$
p_{\chi}(t)=\sum_{i=0}^{\infty} a_{i}(\chi) t^{i}
$$

where $a_{i}(\chi)$ is the multiplicity of $\chi$ in the $i$ th homogeneous component of $K\left[x_{1}, \ldots, x_{n}\right] / I$. (Note that what we call $p_{\chi}(t)$ is $p_{\bar{\chi}}(t)$ in [15], because there $G$ acts on $K\left[x_{1}, \ldots, x_{n}\right]$ via the adjoint representation.) It turns out that $a_{i}(\chi)=0$ if $i>\sum_{j=1}^{n}\left(d_{j}-1\right)$ [15, Lemma 2.9]. The coefficient of $t^{d}$ in the formal power series

$$
\frac{1}{|G|} \sum_{g \in G} \frac{\left(\omega_{1}(g)+\ldots+\omega_{n}(g)\right) \sum_{1 \leq i<j \leq n} \omega_{i}(g) \omega_{j}(g)}{\prod_{i=1}^{n}\left(1-\omega_{i}(g) t\right)}
$$

is the scalar product of the character $\sum_{i=1}^{r} m_{i} \chi_{i}$ and the character of $G$ acting on the $d$ th homogeneous component of $K\left[x_{1}, \ldots, x_{n}\right]$. So by [15, 2.6 Proposition and 2.9 Lemma] we have

$$
H_{1}(t)=\frac{t^{3} \sum_{i=1}^{r} m_{i} p_{\chi_{i}}(t)}{\prod_{i=1}^{n}\left(1-t^{d_{i}}\right)}=\frac{G(t)}{\prod_{i=1}^{n}\left(1-t^{d_{i}}\right)},
$$

where $G(t)$ is of the form

$$
G(t)=\sum_{i=1}^{n\left(\begin{array}{c}
n \\
2
\end{array}\right)} t^{e_{i}}
$$

with $0 \leq e_{1} \leq \ldots \leq e_{n\left(\begin{array}{c}n \\ 2\end{array}\right)} \leq \sum_{i=1}^{n}\left(d_{i}-1\right)+3$.

Now we reduce to the case of irreducible groups as it was done by Guralnick in [9]. Assume that $n \geq 2$ is minimal such that $F_{n}(M)^{G}$ is generated by $n$ elements for some pseudo-reflection group $G$.

1. The same argument as in [9] shows that $G$ is not Abelian.

$[9$, Lemma 3] remains valid with the same proof for any relatively free algebra instead of the generic matrix algebra. Therefore the minimality of $n$ implies that $G$ is irreducible, and we may assume that $G$ is a complex unitary pseudo-reflection group. We shall use the classification of these groups given in [13].

2. $n>2$.

Suppose that $n=2$, and let $\chi=\omega_{1}+\omega_{2}$ denote the character of the given representation of $G$ as a subgroup of $G l_{2}(\mathbb{C})$. We showed above that the degree of $G(t)$ (see (4.2)) is at most $d_{1}+d_{2}+1$. Though this bound would suffice for our purpose, for sake of completeness we derive some more precise information on the polynomial $G(t)$ :

$$
\begin{aligned}
H_{1}(t) & =\frac{1}{|G|} \sum_{g \in G} \frac{\chi(g) \omega_{1}(g) \omega_{2}(g) t^{3}}{\left(1-\omega_{1}(g) t\right)\left(1-\omega_{2}(g) t\right)} \\
& =\frac{1}{|G|} \frac{t^{3}}{t^{2}} \sum_{g \in G} \frac{\chi(g)}{\left(1-\omega_{1}\left(g^{-1}\right) t^{-1}\right)\left(1-\omega_{2}\left(g^{-1}\right) t^{-1}\right)} \\
& =\frac{t}{|G|} \sum_{g \in G} \frac{\bar{\chi}(g)}{\left(1-\omega_{1}(g) t^{-1}\right)\left(1-\omega_{2}(g) t^{-1}\right)} \\
& =t \frac{p_{\chi}\left(t^{-1}\right)}{\left(1-t^{-d_{1}}\right)\left(1-t^{-d_{2}}\right)}
\end{aligned}
$$


by a similar argument we used before. The character of the representation of $G$ on the 1st homogeneous component of $K\left[x_{1}, \ldots, x_{n}\right] / I$ is $\chi$, so $p_{\chi}(t)=t+$ $\sum_{i=2}^{d_{1}+d_{2}-2} a_{i}(\chi) t^{i}$, hence

$$
H_{1}(t)=t \frac{t^{-1}+\sum_{i=2}^{d_{1}+d_{2}-2} a_{i}(\chi) t^{-i}}{\left(1-t^{-d_{1}}\right)\left(1-t^{-d_{2}}\right)}=\frac{t^{d_{1}+d_{2}}+\sum_{j=3}^{d_{1}+d_{2}-1} a_{d_{1}+d_{2}+1-j}(\chi) t^{j}}{\left(1-t^{d_{1}}\right)\left(1-t^{d_{2}}\right)} .
$$

On the other hand, the minimal degree term of $\frac{F(t)}{\prod_{i=1}^{n}\left(1-t^{d_{i}}\right)}$ is $t^{2 d_{1}+d_{2}}$, thus the coefficient of $t^{d_{1}+d_{2}}$ in $D(t)=\frac{F(t)-G(t)}{\left(1-t^{d_{1}}\right)\left(1-t^{d_{2}}\right)}$ is -1 . This contradicts the assumption that $D(t)$ has non-negative coefficients.

3. $G$ is not one of the groups of no. $23,28,30,35,36,37$ in the table [13, Table VII]. (The argument in 5 below applies also for these groups. However, first we eliminate them by exhibiting some invariants. This argument shows the role of the polynomial $\left[x_{2}, x_{1}, x_{1}\right]$ explicitly.)

Suppose that $G$ is one of these groups. It is well known (see for example [16, 4.2.15. Lemma]) that $G$ can be defined over the reals if and only if $G$ has an invariant of degree 2. So we can suppose that $G<G l_{n}(\mathbb{R})$ is an orthogonal group, and then a straightforward calculation shows that

$$
x_{1}^{2}+\ldots+x_{n}^{2} \in K\left\langle x_{1}, \ldots, x_{n}\right\rangle^{G} .
$$

Thus we can choose $f_{1}, \ldots, f_{n}$ such that

$$
f_{1}=y_{1}^{2}+\ldots+y_{n}^{2}
$$

Consider the invariant

$$
f=\sum_{g \in G} g \cdot\left(y_{1}\left(y_{1}^{2}+\ldots+y_{n}^{2}\right) y_{1}\right)
$$

Comparing the condition $K\left\langle f_{1}, \ldots, f_{n}\right\rangle=F_{n}(M)^{G}$ and the degrees of the groups under consideration (cf. [13, Table VII]) we conclude that the only invariants of $G$ of degree 4 are the scalar multiples of $f_{1}^{2}$, therefore $f=a f_{1}^{2}$ in $F_{n}(M)$ for some $a \in \mathbb{R}$. Now we have

$$
f=\sum_{g \in G}\left(g_{11} y_{1}+\ldots+g_{n 1} y_{n}\right)\left(y_{1}^{2}+\ldots+y_{n}^{2}\right)\left(g_{11} y_{1}+\ldots+g_{n 1} y_{n}\right),
$$

and the polynomial

$h=\sum_{g \in G}\left(g_{11} x_{1}+\ldots+g_{n 1} x_{n}\right)\left(x_{1}^{2}+\ldots+x_{n}^{2}\right)\left(g_{11} x_{1}+\ldots+g_{n 1} x_{n}\right)-a\left(x_{1}^{2}+\ldots+x_{n}^{2}\right)^{2}$

is contained in $M$. The homogeneous component of $h$ of degree 4 in $x_{i}$ is

$$
\left(\sum_{g \in G} g_{i 1}^{2}-a\right) x_{i}^{4}, \quad(i=1, \ldots, n)
$$


and it is contained in $M$, implying that $a=\sum_{g \in G} g_{i 1}^{2}, i=1, \ldots, n$. Since for any $g \in G$ there exists an $i$ with $g_{i 1} \neq 0$, we have $a \neq 0$. The multihomogeneous component of $h$ of multidegree $(2,2)$

$\sum_{g \in G} g_{11}^{2} x_{1} x_{2}^{2} x_{1}+\sum_{g \in G} g_{21}^{2} x_{2} x_{1}^{2} x_{2}-a x_{1}^{2} x_{2}^{2}-a x_{2}^{2} x_{1}^{2}=a\left(x_{1} x_{2}^{2} x_{1}+x_{2} x_{1}^{2} x_{2}-x_{1}^{2} x_{2}^{2}-x_{2}^{2} x_{1}^{2}\right)$

is contained in $M$. Make the substitution $x_{1} \rightarrow x_{1}+1, x_{2} \rightarrow x_{2}+1$ in the above polynomial, then we get

$$
2 a x_{1} x_{2} x_{1}+\text { other monomials. }
$$

Hence $M$ contains a polynomial of degree 3 , which contradicts the definition of $M$.

4. $G$ is not $G(m, p, n)$ (the groups no. 2 in [13, Table VII]).

Recall the definition of $G(m, p, n)$. Let $n \geq 3$ (the case $n=2$ was handled in 2.), $m \geq 2, p$ be positive integers such that $p$ divides $m$. Let $A(m, p, n)<G l_{n}(\mathbb{C})$ be the group of diagonal matrices whose diagonal entries are $m$ th roots of unity and the determinant is an $\frac{m}{p}$ th root of unity. Consider $\operatorname{Sym}(n)$ as the group of permutation matrices in $G l_{n}(\mathbb{C})$. Clearly, $\operatorname{Sym}(n)$ normalizes $A(m, p, n)$, and $G(m, p, n)$ is defined as the semidirect product

$$
G(m, p, n)=A(m, p, n) \rtimes \operatorname{Sym}(n) .
$$

Consider the polynomials

$$
\begin{gathered}
h_{i}=\sum_{\pi \in \operatorname{Sym}(n)} \pi \cdot \sigma_{i}\left(x_{1}^{m}, \ldots, x_{n}^{m}\right), \quad i=1, \ldots, n-1 ; \\
h_{n}=\sum_{\pi \in \operatorname{Sym}(n)} \pi \cdot\left(x_{1} \ldots x_{n}\right)^{m / p} .
\end{gathered}
$$

The above semidirect decomposition of $G(m, p, n)$ shows that

$$
h_{1}, \ldots, h_{n} \in K\left\langle x_{1}, \ldots, x_{n}\right\rangle^{G},
$$

and it is well known that their images in $K\left[x_{1}, \ldots, x_{n}\right]$ form a basic set of invariants, with degrees $m, 2 m, 3 m, \ldots,(n-1) m, \frac{m}{p} n$. So we may suppose that $f_{1}, \ldots, f_{n}$ are the images of $h_{1}, \ldots, h_{n}$ under the natural homomorphism $K\left\langle x_{1}, \ldots, x_{n}\right\rangle \rightarrow$ $F_{n}(M)$. Consider the invariant

$$
f=\sum_{g \in G} g \cdot\left(y_{1}^{m-1} f_{1} y_{1}\right)
$$

Any $g \in G$ can be written in the form $g=a \pi$ for some $a \in A(m, p, n)$ and $\pi \in \operatorname{Sym}(n)$. So we have $g\left(y_{1}\right)=\theta y_{\pi(1)}$ for some permutation $\pi$ and $m$ th root of unity $\theta$, and

$$
f=(n-1) ! m^{n} / p \sum_{i=1}^{n} y_{i}^{m-1}\left(y_{1}^{m}+\ldots+y_{n}^{m}\right) y_{i} .
$$


By our hypothesis $f$ is contained in $K\left\langle f_{1}, \ldots, f_{n}\right\rangle$, and since $\operatorname{deg}\left(f_{3}\right), \ldots, \operatorname{deg}\left(f_{n}\right)>$ $\operatorname{deg}(f)=2 m, f$ can be expressed with $f_{1}, f_{2}$ and $f_{n}$. More precisely, there exists a polynomial

$$
h=\sum_{i=1}^{n} x_{i}^{m-1}\left(x_{1}^{m}+\ldots+x_{n}^{m}\right) x_{i}-\left(c_{1} h_{1}^{2}+c_{2} h_{2}+\sum_{j} a_{j} h_{n} b_{j}\right) \in M,
$$

where $c_{1}, c_{2} \in \mathbb{C}$, and $a_{j}, b_{j} \in K\left\langle x_{1}, \ldots, x_{n}\right\rangle$. Consider the multihomogeneous components of $h$. The coefficient of $x_{1}^{2 m}$ is $1-c_{1}$, implying that $c_{1}=1$. Since $n \geq 3$, any monomial of $h_{n}$ contains the variable $x_{3}$, therefore the sum of the coefficients of the monomials of multidegree $(m, m)$ is $2-2 c_{1}-k c_{2}$ for some positive integer $k$, showing that $c_{2}=0$. Thus the polynomial

$$
x_{1}^{m-1} x_{2}^{m} x_{1}+x_{2}^{m-1} x_{1}^{m} x_{2}-x_{1}^{m} x_{2}^{m}-x_{2}^{m} x_{1}^{m}
$$

is contained in $M$. After the substitution $x_{1} \rightarrow x_{1}+1, x_{2} \rightarrow x_{2}+1$ we obtain a polynomial in which the coefficient of $x_{1} x_{2} x_{1}$ is $(m-1) m$. Again this contradicts the fact that $M$ has no elements of degree 3 .

5. $G$ is not one of the groups no. $24,26,27,29,31,32,33,34$ or no. 1 with $n \geq 4$ in [13, Table VII].

Let $a, b$ be functions $\mathbb{N} \rightarrow \mathbb{C}$, and let $c$ be a function $\mathbb{N} \rightarrow \mathbb{N}$. We say that $a(n)=b(n)+O(c(n))$, if $|a(n)-b(n)| \leq L c(n)$ for some constant $L$. We need the following lemma (cf. [16, 2.5.9. Lemma]):

Lemma 4.1. Let $H(t) \in \mathbb{C}[[t]]$ be a formal power series of the form

$$
H(t)=\frac{A(t)}{\prod_{i=1}^{n}\left(1-t^{d_{i}}\right)}=\sum_{d=0}^{\infty} c_{d} t^{d},
$$

where $A(t) \in \mathbb{C}[t]$.

$$
\left.(1-t)^{n} H(t)\right|_{t=1}=\frac{A(1)}{\prod_{i=1}^{n} d_{i}}
$$

$$
(1-t)^{n-1} H(t)-\left.\frac{A(1)}{\prod_{i=1}^{n} d_{i}} \frac{1}{1-t}\right|_{t=1}=\frac{\frac{1}{2} A(1) \sum_{i=1}^{n}\left(d_{i}-1\right)-A^{\prime}(1)}{\prod_{i=1}^{n} d_{i}} .
$$

(iii) Suppose that $n \geq 3$ and all sets of $n-1$ of the $d_{i}$ have greatest common divisor 1. Then we have

$$
c_{d}=\frac{A(1)}{(n-1) ! \prod_{i=1}^{n} d_{i}} d^{n-1}+\frac{\frac{1}{2} A(1) \sum_{i=1}^{n} d_{i}-A^{\prime}(1)}{(n-2) ! \prod_{i=1}^{n} d_{i}} d^{n-2}+O\left(d^{n-3}\right) .
$$

(In [16] $n \geq 4$ is required, but the proof works also for $n=3$.)

Now we investigate the power series $D(t)=\frac{F(t)-G(t)}{\prod_{i=1}^{n}\left(1-t^{d_{i}}\right)}$. Since $F(1)=G(1)=$ $n\left(\begin{array}{l}n \\ 2\end{array}\right)$, by Lemma 4.1 (i) $D(t)$ has no pole $\frac{1}{(1-t)^{n}}$. By Lemma 4.1 (ii) the coefficient of the pole $\frac{1}{(1-t)^{n-1}}$ in the Laurent series of $\frac{F(t)}{\prod_{i=1}^{n}\left(1-t^{d_{i}}\right)}$ is

$$
\frac{\frac{1}{2} n\left(\begin{array}{l}
n \\
2
\end{array}\right) \sum_{i=1}^{n}\left(d_{i}-1\right)-\frac{3}{2} n(n-1) \sum_{i=1}^{n} d_{i}}{\prod_{i=1}^{n} d_{i}}=\frac{n(n-1)}{4|G|}\left((n-6) \sum_{i=1}^{n}\left(d_{i}-1\right)-6 n\right) .
$$


By (4.1) the coefficient of the pole $\frac{1}{(1-t)^{n-1}}$ in the Laurent series of $H_{1}(t)$ is

$$
\frac{1}{|G|} \sum_{g \in R} \frac{(1+\ldots+1+\omega(g))\left(\left(\begin{array}{c}
n-1 \\
2
\end{array}\right)+(n-1) \omega(g)\right)}{1-\omega(g)},
$$

where $R=\{g \in G \mid g$ is a pseudo-reflection $\}$ and $\omega(g)$ is the eigenvalue of $g$ different from 1. It is well known (and one can derive from Lemma 4.1 (i)) that

$$
\sum_{g \in R} \frac{1}{1-\omega(g)}=\frac{1}{2} \sum_{i=1}^{n}\left(d_{i}-1\right)
$$

Using this formula we get the equalities

$$
\sum_{g \in R} \frac{\omega(g)}{1-\omega(g)}=-\sum_{g \in R} \frac{1}{1-\bar{\omega}(g)}=-\frac{1}{2} \sum_{i=1}^{n}\left(d_{i}-1\right)
$$

and

$$
\sum_{g \in R} \frac{\omega(g)^{2}}{1-\omega(g)}=\sum_{g \in R}\left(-1-\omega(g)+\frac{1}{1-\omega(g)}\right)=N-\frac{1}{2} \sum_{i=1}^{n}\left(d_{i}-1\right),
$$

where $N$ is the number of the reflecting hyperplanes. Thus we have

$$
\begin{aligned}
\frac{1}{|G|} \sum_{g \in R} \frac{((n-1)+\omega(g))\left(\left(\begin{array}{c}
n-1 \\
2
\end{array}\right)+(n-1) \omega(g)\right)}{1-\omega(g)} & \\
= & \frac{n-1}{4|G|}\left(\left(n^{2}-6 n+4\right) \sum_{i=1}^{n}\left(d_{i}-1\right)+4 N\right),
\end{aligned}
$$

and the coefficient of the pole $\frac{1}{(1-t)^{n-1}}$ in $D(t)$ is

$$
\frac{n-1}{2|G|}\left(-3 n^{2}-2 \sum_{i=1}^{n}\left(d_{i}-1\right)-2 N\right)=\frac{G^{\prime}(1)-F^{\prime}(1)}{|G|} .
$$

(The last equality holds by Lemma 4.1 (ii).)

Denote by $\delta$ the greatest common divisor of $d_{1}, \ldots, d_{n}$. The assumption $F_{n}(M)^{G}$ $=K\left\langle f_{1}, \ldots, f_{n}\right\rangle$ implies that $\delta$ divides each of the $e_{1}, \ldots, e_{n\left(\begin{array}{c}n \\ 2\end{array}\right)}$, that is, we may write

$$
D(t)=\frac{A(s)}{\prod_{i=1}^{n}\left(1-s^{d_{i} / \delta}\right)}=\sum_{d=0}^{\infty} c_{d} s^{d}
$$

where $s=t^{\delta}$ and $A(s) \in \mathbb{C}[s]$. One can check in [13, Table VII] that for the groups under consideration the numbers $d_{1} / \delta, \ldots, d_{n} / \delta$ satisfy the condition of Lemma 4.1 (iii), so applying this lemma and the equalities $A(1)=0$ and $\left.\frac{\partial}{\partial s} A(s)\right|_{s=1}=$ $\delta\left(G^{\prime}(1)-F^{\prime}(1)\right)$ we obtain

$$
\begin{aligned}
c_{d} & =\frac{\delta\left(G^{\prime}(1)-F^{\prime}(1)\right)}{(n-2) ! \prod_{i=1}^{n} d_{i}} d^{n-2}+O\left(d^{n-3}\right) \\
& =\frac{-\delta(n-1)}{2(n-2) !|G|}\left(3 n^{2}+2 \sum_{i=1}^{n}\left(d_{i}-1\right)+2 N\right) d^{n-2}+O\left(d^{n-3}\right) .
\end{aligned}
$$


This immediately implies that for sufficiently large $d$ the coefficient of $t^{d}$ in the power series $D(t)$ is strictly negative, which is a contradiction.

6. $G$ is not the group no. 25 in [13, Table VII].

This group has 24 pseudo-reflections, all of them are of order 3 (see [3, p. 412, Table]), so the number of reflecting hyperplanes is 12 . By the calculations in 5 we have

$$
F^{\prime}(1)-G^{\prime}(1)=3 \cdot 3^{2}+2 \cdot 24+2 \cdot 12 \text {. }
$$

On the other hand

$$
F^{\prime}(1)-G^{\prime}(1)=9\left(d_{1}+d_{2}+d_{3}\right)-\left(e_{1}+\ldots+e_{9}\right)
$$

therefore

$$
\frac{e_{1}+\ldots+e_{9}}{9}=16
$$

implying that $e_{1} \leq 16$. But we have

$$
F(t)=t^{2 d_{1}+d_{2}}+\text { higher degree terms, }
$$

and since $2 d_{1}+d_{2}=21$, the coefficient of $t^{e_{1}}$ in $D(t)=\frac{F(t)-G(t)}{\prod_{i=1}^{3}\left(1-t^{d_{i}}\right)}$ is strictly negative. This is a contradiction.

7. $G$ is not $\operatorname{Sym}(4)$, that is, the group no. 1 with $n=3$ in [13, Table VII].

One can calculate the power series $D(t)$ directly and conclude that it has a negative coefficient.

We have eliminated all the finite irreducible complex unitary reflection groups, so the proof of Proposition 2.4 is complete.

\section{ACKNOWLEDGEMENTS}

I would like to thank the Széchenyi István Scholarship Foundation for the support which made it possible to visit the Penn State University in the first half of 1994. I thank the Department of Mathematics, especially E. Formanek for their hospitality during my visit. I am also grateful to V. Drensky for some earlier conversations on this topic.

\section{REFERENCES}

1. N. Bourbaki, Groupes et Algébres de Lie, Chap. IV, V, VI, Hermann, Paris, 1968. MR 39:1590

2. C. Chevalley, Invariants of finite groups generated by reflections, Amer. J. Math. 77 (1955), 778-782. MR 17:345d

3. A. M. Cohen, Finite complex reflection groups, Ann. Sci. École Norm. Sup. 9 (1976), 379-436. MR 54:10437

4. W. Dicks and E. Formanek, Poincare series and a problem of S. Montgomery, Lin. Mult. Alg. 12 (1982), 21-30. MR 84c: 15032

5. V. Drensky, Polynomial identities for $2 \times 2$ matrices, Acta Appl. Math. 21 (1990), 137-161. MR 91k:16024

6. V. Drensky, Computational techniques for PI-algebras, Topics in Algebra, Banach Center Publications, Vol. 26, Part 1., PWN-Polish Scientific Publishers, Warsaw, 1990, pp. 17-44. MR 93e: 16036

7. V. Drensky, Private communication (1993).

8. E. Formanek, Noncommutative invariant theory, Contemporary Mathematics 43, Group Actions on Rings, S. Montgomery ed., Amer. Math. Soc., 1985, pp. 87-119. MR 87d:16046 
9. R. M. Guralnick, Invariants of finite linear groups acting on relatively free algebras, Linear Alg. Appl. 72 (1985), 85-92. MR 87d:16050

10. V. K. Kharchenko, Noncommutative invariants of finite groups and Noetherian varieties, J. Pure Appl. Alg. 31 (1984), 83-90. MR 85j:16052

11. D. Krakowsky and A. Regev, The polynomial identities of the Grassmann algebra, Trans. Amer. Math. Soc. 181 (1973), 429-438. MR 48:4005

12. L. H. Rowen, Polynomial Identities in Ring Theory, Academic Press, New York, 1980. MR 82a: 16021

13. G. C. Shephard and J. A. Todd, Finite unitary reflection groups, Canad. J. Math. 6 (1954), 274-304. MR 15:600b

14. L. Solomon, Invariants of finite reflection groups, Nagoya Math. J. 22 (1963), 57-64. MR 27:4872

15. T. A. Springer, Regular elements of finite reflection groups, Inv. Math. 25 (1974), 159-198. MR 50:7371

16. T. A. Springer, Invariant Theory, Lecture Notes in Mathematics 585, Springer-Verlag, Berlin, Heidelberg, New York, 1977. MR 56:5740

Mathematical Institute of the Hungarian Academy of Sciences, P.O.B. 127, H-1364, Budapest, Hungary

E-mail address: domokos@math-inst.hu 